University of Nebraska - Lincoln

DigitalCommons@University of Nebraska - Lincoln

July 2002

\title{
Band structure of strained $\mathrm{Gd}(0001)$ films
}

\author{
I. N. Yakovkin \\ University of Nebraska-Lincoln \\ Takashi Komesu \\ University of Nebraska-Lincoln, tkomesu2@unl.edu \\ Peter A. Dowben \\ University of Nebraska-Lincoln, pdowben@unl.edu
}

Follow this and additional works at: https://digitalcommons.unl.edu/physicsdowben

Part of the Physics Commons

Yakovkin, I. N. ; Komesu, Takashi; and Dowben, Peter A., "Band structure of strained Gd(0001) films" (2002). Peter Dowben Publications. 18.

https://digitalcommons.unl.edu/physicsdowben/18

This Article is brought to you for free and open access by the Research Papers in Physics and Astronomy at DigitalCommons@University of Nebraska - Lincoln. It has been accepted for inclusion in Peter Dowben Publications by an authorized administrator of DigitalCommons@University of Nebraska - Lincoln. 


\title{
Band structure of strained Gd(0001) films
}

\author{
I. N. Yakovkin, ${ }^{1}$ Takashi Komesu, ${ }^{2}$ and P. A. Dowben ${ }^{2}$ \\ ${ }^{1}$ Institute of Physics, National Academy of Sciences of Ukraine, Prospect Nauki 46, Kiev 03028, Ukraine \\ ${ }^{2}$ Department of Physics and Astronomy and the Center for Materials Research and Analysis, Brace Laboratory 116,
} P.O. Box 880111, University of Nebraska, Lincoln, Nebraska 68588-0111

(Received 7 February 2002; published 12 July 2002)

\begin{abstract}
The electronic structure of strained and unstrained $\mathrm{Gd}(0001)$ surfaces has been studied both theoretically and experimentally with spin-polarized photoemission spectroscopy and spin-polarized inverse photoemission spectroscopy. Good agreement between calculated surface bands and surface-induced features of the spectra provides the basis for a more detailed explanation of the origin of the spin-polarized bands than was previously possible. It has been found that observed relaxation of the expansively strained in-plane crystal lattice constant, of $\mathrm{Gd}(0001)$ on $\mathrm{Mo}(112)$, significantly affects the electronic structure of the surface.
\end{abstract}

DOI: 10.1103/PhysRevB.66.035406

PACS number(s): 75.25.+z; 75.80.+q; 71.20. $-\mathrm{b} ; 71.70 .-\mathrm{d}$

\section{INTRODUCTION}

Strain is known to affect magnetism, with possible dramatic effects as suggested by the theoretical calculations of Moruzzi and Marcus ${ }^{1}$ and experimental results of Shinde et al., ${ }^{2}$ Bartholin et al., ${ }^{3}$ and others. There is a general acceptance of the strong influence of magnetoelastic interactions on the Curie temperature and other magnetic properties. For rare-earth metals, the magnetoelastic interactions are large. ${ }^{3-6}$ Compression of gadolinium is seen to lead to a suppression of $T_{c},{ }^{3,6}$ while expansion leads to an increase of $T_{c}{ }^{7,8}$ Not only does strain affect the magnetic properties, as now detailed fairly extensively for the perovskites, ${ }^{9-17}$ but it has long been established that the lattice constant has a profound influence on the electronic structure, ${ }^{18-20}$ even for the thinnest of thin films. ${ }^{21-26}$

Gd(0001) grown on Mo(112) exhibits a substantial inplane expansive strain compared to a similar thickness of $\mathrm{Gd}(0001)$ on $\mathrm{W}(110) .^{21}$ For $\mathrm{Gd}$ grown on $\mathrm{Mo}(112)$, the lattice is expanded by $4 \%$ for a film thickness of 30 to $150 \AA \AA^{21}$ The expansive $4 \%$ in-plane strained $\mathrm{Gd}(0001)$ on $\mathrm{Mo}(112)$ results in a quite different electronic structure and altered magnetic properties compared to the strain relieved $\mathrm{Gd}(0001)$ grown on $\mathrm{W}(110),{ }^{27}$ as noted elsewhere. ${ }^{8,21,28,29}$ Based on studies of rare-earth alloys, Andrianov ${ }^{19,20}$ suggested that the Fermi surface is sensitive to both composition and lattice deformation. It was shown ${ }^{28}$ that the relief of the strain in $\mathrm{Gd}(0001)$ on $\mathrm{Mo}(112)$ with increasing film thickness results in a spin-polarized electronic structure that is increasingly similar to the largely unstrained $\operatorname{Gd}(0001)$ films grown on $\mathrm{W}(110)$.

$\mathrm{Gd}(0001)$ grown on a $\mathrm{W}(110)$ surface has been heavily investigated over the past decade. ${ }^{30}$ For $\operatorname{Gd}(0001)$ grown on $\mathrm{W}(110)$, the hexagonal-close-packed (hcp) film has been observed to be strained $(2-3 \%)$ for a film thickness of $10-50$ $\AA .{ }^{27}$ Gd grown on W(110) then relaxes toward a bulk lattice constant with increasing film thickness, so that with sufficient deposition of $\mathrm{Gd}$, the bulk $\mathrm{Gd}$ lattice parameter (3.63 $\AA$ ) is reached at $100-1000 \AA .{ }^{27}$ While it is now clear that the $\mathrm{Gd}(0001)$ films on $\mathrm{Mo}(112)$, for a thickness less than $40 \mathrm{ML}$, are substantially more strained than $\mathrm{Gd}(0001)$ overlayers on $\mathrm{W}(110),{ }^{27}$ the mechanism of strain relief with increasing film thickness which is observed for $\mathrm{Gd}(0001)$ on $\mathrm{W}(110)$ (Ref. 27) should be applicable to $\mathrm{Gd}(0001)$ on $\mathrm{Mo}(112){ }^{28}$

While a number of experimental studies have been undertaken to investigate the spin-polarized band structure of strained $\operatorname{Gd}(0001),{ }^{7,8,29}$ an interpretation of experimental data is complicated by the close proximity of bulk and surface bands, and the large number of observed unoccupied bands, not seen with $\mathrm{Gd}(0001)$ on $\mathrm{W}(110))^{30-32}$ A great interest in the behavior of the surface and bulk band structure of gadolinium has provoked a number of theoretical investigations of the $\mathrm{Gd}(0001)$ surface using various calculational techniques and approximations for exchange-correlation potential. ${ }^{33-45}$ Nevertheless, mostly due to biased treatment of $4 f$ electrons, the calculations (despite the diversity of approaches) have exhibited only limited agreement with experiment. In particular, to date, there is no convincing explanation for the spectral features observed for strained $\mathrm{Gd}(0001)$ surface, while for unstrained surface only a qualitative agreement, at best, with angle-resolved photoemission (ARPES) data has been achieved.

The objective of the present paper is to reconcile some of the differences between the calculated band structures and results of spin- and angle-resolved photoemission and spinand angle-resolved inverse photoemission studies for the strained and unstrained $\operatorname{Gd}(0001)$ surfaces. With this aim, we perform a detailed comparison of theoretical surface and bulk band structures, with experimental data obtained by spin-polarized photoemission (SPES) and spin-polarized inverse photoemission (SPIPES) (partly published elsewhere ${ }^{8,29}$ ). The band structures have been calculated by the linear augmented-plane-wave (LAPW) method for thin films. ${ }^{46-49}$

\section{EXPERIMENT}

We have mapped out the spin-polarized band structure of thin Gd films grown on the Mo(112) surface along the $\bar{\Gamma}-\bar{M}$ high-symmetry direction of the surface Brillouin zone. Spinpolarized inverse photoemission spectra were obtained in an ultrahigh-vaccum system in the isochromatic mode $(\hbar \omega=9.4$ $\pm 0.3 \mathrm{eV}$ ) with a Geiger-Müller tube and a spin-polarized electron gun based on the Ciccacci design (with a GaAs 
photocathode). ${ }^{50}$ The results were complemented by SPES experiments carried out at the U5A undulator beamline of the National Synchrotron Light Source (NSLS) at Brookhaven National Laboratory. The details of the experimental setup(s) are described elsewhere. ${ }^{51}$

The clean $\operatorname{Mo}(112)$ surface was obtained after the standard surface preparation treatment which included heating in oxygen atmosphere with subsequent serial flashing to the temperatures above $2100 \mathrm{~K}$ to remove the oxygen contamination. The $\operatorname{Gd}(0001)$ films were grown at room temperature at the base pressure of $7 \times 10^{-11}$ Torr and subsequently annealed. The crystal quality of the $\mathrm{Gd}(0001)$ films was determined by low-energy electron diffraction, which was also used to determine the extent of the expansive strain. The experimental band dispersion, from both spin-polarized photoemission and spin-polarized inverse photoemission, was used to confirm the presence of strain from the position of the surface Brillouin zone critical points. ${ }^{21,29}$

\section{THEORETICAL METHODOLOGY}

The band structures of bulk Gd with hep and fcc structures and for a single slab of 5-ML thickness to simulate both surface and bulk contributions, were calculated by the scalar relativistic all-electron linearized augmented plane-wave (LAPW) method for thin films. ${ }^{45-48}$ The self-consistent potential was recalculated for each iteration taking into account the redistribution of all core electrons. The number of basis functions was adjusted to provide mRy convergence for the bands near $E_{F}$. Bulk and surface densities of states (DOS's) were calculated using the tetrahedron or triangular integration method, respectively. ${ }^{49}$

The LAPW method is widely recognized, and spinpolarized band-structure calculations would be routine if one could accurately treat exchange-correlation effects. It has been established that the local-spin-density approximation (LSDA) fails to describe the electronic structure of strongly correlated systems such as Mott insulators, insulator ferromagnets, and $4 f$ metals correctly. ${ }^{33,38,44}$ The inability of the LSDA to correctly describe $4 f$ metals occurs mainly because of incorrect position of spin-up $f_{\uparrow}$ and spin-down $f_{\downarrow}$ bands if the $4 f$ electrons are treated as bands (not as core states). ${ }^{34-36}$

Harmon and Freeman ${ }^{37}$ calculated the band structure for Gd by the augmented plane-waves method using the exchange-correlation $X_{\alpha}$ potential with two values, i.e., $\alpha$ $=1$ ("total Slater's exchange") and $\alpha=2 / 3$ ("Kohn-Sham potential"). In this pioneering work, the occupied $4 f_{\uparrow}$, states were treated as core states and given, following Hund's rule, a rigid value of magnetic moment $\mu=7 \mu_{B}$ associated with spin-up $4 f^{7}$ electrons. In order to get the experimental value of $7.55 \mu_{B}$ for $\mathrm{Gd}$, the rest magnetic moment, some $0.55 \mu_{B}$, was provided by properly choosing the configuration of the $5 d 6 s$ valence electrons (these calculations were not selfconsistent). The results for $\alpha=1$ were in reasonable agreement with experiment, though obtained width of the valence band (from the bottom at $\Gamma_{1}$ to $E_{F}$ ) of $3.1 \mathrm{eV}$ seems too small comparatively to derived from photoemission spectroscopy value of about $4 \mathrm{eV}$.
Later, Harmon et al. ${ }^{38}$ and Singh ${ }^{44}$ suggested that correct total energies for Gd could not be obtained if $4 f$ electrons were treated as core states. Instead, a claim was made that these states must be considered as band states, while inconsistencies of the energies of these calculated states with experiment were explained by a failure of the LSDA. The increasingly compelling evidence that gadolinium was a local moment system with correlated electrons ${ }^{30}$ provoked the development of improved version of the LSDA by including the Hubbard $U$ method, taken from the mean-field approach. ${ }^{33}$ To date, the LSDA $+\mathrm{U}$ method is widely recognized and has proved fruitful in recent calculations of the bulk and surface electronic structure of $\mathrm{Gd}^{41-43}$ Due to a better accounting of the intra-atomic correlations, the $4 f$ minority band rises away from the Fermi level while the $4 f$ majority band increases in binding energy to the correct position $^{41}$ [approximately $-8.5 \mathrm{eV}$ with respect to $E_{F}$ (Ref. 30)]. Implementation of the LSDA+U method into the LAPW method for thin films has been accomplished, ${ }^{42}$ and calculated self-consistent densities of states for $\mathrm{Gd}(0001)$ surface provide a reasonable positioning of the occupied $4 f$ band. Again, inclusion of the Hubbard U allowed for a correct estimation of the magnetic moment as well as of the width of the $s$ - $d$ valence band (about $4 \mathrm{eV}$ ). ${ }^{43}$

On the other hand, the LSDA $+\mathrm{U}$ results ultimately predict the existence of the spin-minority unoccupied band approximately at $+2 \mathrm{eV}$ above $E_{F}$. This band originates from the Gd $4 f$ minority localized "atomic" state and therefore is very narrow [0.5 eV (Refs. 41 and 43)] thus it results in an extremely high peak in unoccupied DOS's [the "pure" LSDA approach puts this peak onto $E_{F}$ (Ref. 41)]. The challenge here is that for the Gd surface, such a peak is very difficult to observe in angle resolved inverse photoemission, ${ }^{52}$ and the existing assignment cannot be completely definitive.

An alternative approach to rare earths, with consideration of $4 f$ states as core states, has been argued ${ }^{45}$ to be relevant, due to a strong localization of the $4 f$ electrons within the $\mathrm{Gd}$ core. Thus Wu et al. ${ }^{45}$ using von Barth and Hedin's exchange-correlation potential, calculated the $\mathrm{Gd}(0001)$ band structure by the FLAPW method. The $4 f^{7}$ electrons were treated as core states, which canceled the problem of the "ghost" $4 f$ minority band. Despite the overly large width of the occupied $s-d$ band (almost $6 \mathrm{eV}$ ), the results are in qualitative agreement with angle-resolved photoemission data. ${ }^{53-55}$ In particular, the calculations reveal the existence of the spin majority $-0.3 \mathrm{eV}$ (in the vicinity of $\bar{\Gamma}$ ) surface band of $d_{3 z^{2}-r^{2}}$ symmetry ${ }^{53,54}$ as well as spin-minority surface resonance bands above $E_{F}$ which are observed in the spin-polarized photoemission ${ }^{56}$ and spin-polarized inverse photoemission $^{31}$ spectra, respectively. The issue of correct description of $4 f$ states was addressed by Eriksson et al. ${ }^{36} \mathrm{It}$ was suggested that the "first-principles" (pure) LSDA approach can be rescued and resuscitated, provided that the $4 f$ states are treated as core states.

Bylander and Kleinman, ${ }^{34,35}$ in considering $4 f$ states, proposed that the key to the problem is that exchange and correlation should be treated differently for core states and for 

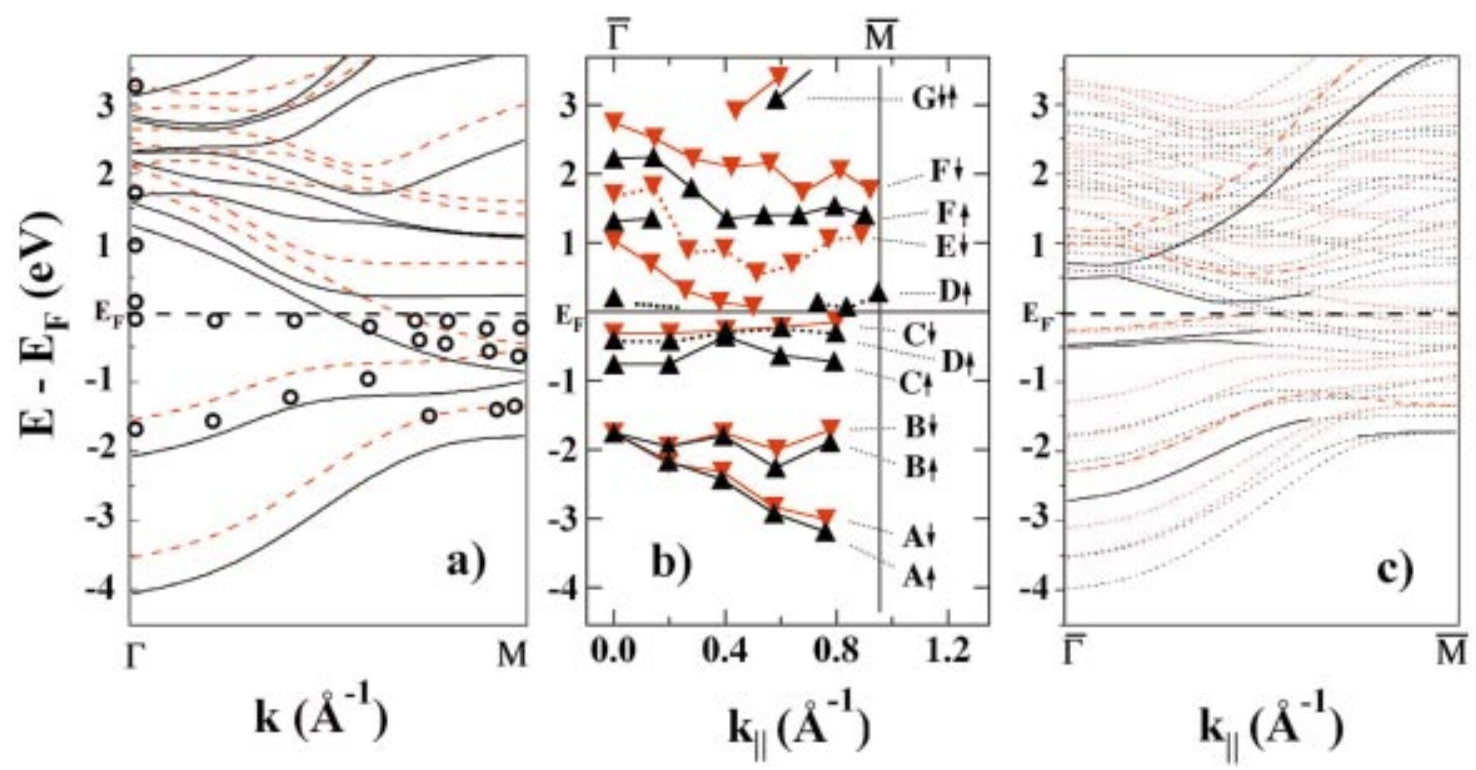

FIG. 1. (Color.) The bulk and surface band structures of gadolinium. The bulk band of unstrained Gd(0001) is shown in (a) with spin-minority (dashed line) and spin-majority (solid line) along $\Gamma M$ symmetry direction of bulk Brillouin zone. The experimental results of spin integrated angle-resolved photoemission (Refs. 53 and 55) and inverse photoemission (Ref. 52) are indicated by circles in (a), for comparison. The experimental band structure of $4 \%$ strained Gd(0001) surface is shown in (b) (in part, adapted from Ref. 29). In (b), downward and upward triangles refer to spin-up (majority) and spin-down (minority) band characters, respectively. The theoretical band structure (c) along the $\bar{\Gamma}-\bar{M}(\bar{\Sigma}$ symmetry line in the surface Brillouin zone), calculated for a five-layer slab of Gd(0001), with $4 \%$ expansive strained lattice, provides a comparison with experiment. Surface states (SS) and surface resonances (SR) for spin-majority and minority electrons are marked by solid and dashed lines. True surface states, with more than $90 \%$ of the charge in the outmost surface layer, appear in vicinity of $\bar{\Gamma}$ in the relative gap in projected bulk band structure.

bands. Thus, the LSDA must be adequate for valence electrons in the band states, while for core electrons the HartreeFock approximation seems more appropriate. It should also be mentioned that authors have questioned whether the $\mathrm{LSDA}+\mathrm{U}$ approach is able to provide a correct electronic structure of Gd. We note that the " $4 f$ as core states" approach involves certain parameters which are difficult to evaluate rigorously. On the other hand, the latter method is not only simpler from a computation point of view, but also avoids the above-mentioned problems of the LSDA+ U approach, and provides quite a reasonable band structure for $\mathrm{Gd}$ - the $s-d$ bandwidth of $4.2 \mathrm{eV}$ and no narrow $4 f$ minority bands just above $E_{F} \cdot{ }^{34}$ Hence this method seems the most attractive approach for purposes of our present study.

Following the methodology of Harmon and Freeman, ${ }^{37}$ we treated the core $4 f^{7}$ shell as completely spin (up) polarized, and thus providing a fixed magnetic moment of $7 \mu_{B}$. We do not consider the chosen methodology as a "firstprinciples" approach, and, following Bylander and Kleinman, ${ }^{34}$ we adopt the parameter description for correlation energy, while the core electrons are treated within the same approximation for the exchange-correlation potential. Obviously, properly determined parameters of the exchangecorrelation potential should yield a correct value of magnetic moment per Gd atom. This requirement has been used in the adjustment of the parameter $\alpha_{2}$ for correlation potential taken in the $\rho^{1 / 3}$ form. Thus the self-consistent band structure for hcp Gd [Fig. 1, left panel (a)], obtained with $\alpha_{1}=0.8$ for exchange (for electrons with the same spin orientation) and $\alpha_{2}=0.2$ for correlation (opposite spins) potential parameters, is in a good agreement with results of band calculations by the mixed-basis pseudopotential method (Bylander and Kleinman $^{34}$ ). Derived from the difference in occupation of the states for majority- and minority-spin electrons, $s-d$ band polarization yields a $0.57 \mu_{B}$ contribution to the total moment. The net calculated magnetic moment per atom is also in good agreement with experiment, and is of order $7.57 \mu_{B}$.

With chosen parameters, the adopted description of exchange-correlation potential seems reasonable and can be used for a LSDA self-consistent all-electron calculation for the surface band structure. No further variations of the form of density functional, such as the $G W$ approximation, ${ }^{57}$ or attempts to improve the local approximation by including density gradient corrections (the generalized gradient approximation) (Ref. 58) were undertaken. Such corrections might be essential for correct evaluations of total energy and ground (ferromagnetic) state, ${ }^{39}$ and are beyond the scope of present study as well as are believed to be unimportant to these band structure calculations.

\section{RESULTS AND DISCUSSION}

\section{A. fec or hep?}

While Gd films, with thickness above $10 \mathrm{ML}$ on Mo(112), have shown to adopt a hexagonal surface structure with about $4 \%$ larger in-plane lattice constant, ${ }^{21}$ compared to that 
of unstrained $\mathrm{Gd}(0001)$ on $\mathrm{W}(110),{ }^{30}$ it is difficult to distinguish such a hexagonal surface structure as corresponding to either fcc (111) or hcp (0001) in either case. Band structure calculations performed for both fcc and hcp Gd illustrate that in spite of similar bandwidths and relative location of $d$ bands, there is a dramatic distinction between band structures for hcp and fcc Gd. This is clear in the appearance (due to two atoms in the Gd hcp unit cell) of the second band (-2 $\mathrm{eV}$ at $\Gamma$ ) for the hcp Gd structure [shown in the left panel (a) of Fig. 1] while it is absent for the calculated band structure of fcc Gd. This band is also evident in experiment [the bands marked $B$ in the central panel (b) of Fig. 1, and the experimental data for unstrained $\operatorname{Gd}(0001)$, plotted on top of the calculated Gd band structure in the left panel (a) of Fig. 1]. Thus adoption of the fcc $\operatorname{Gd}(111)$ structure on the Mo(112) and $\mathrm{W}(110)$ surfaces, during film growth, can be excluded. The hexagonal film structure, found from low-energy electron-diffraction studies, indeed should be attributed to $4 \%$ strained $\mathrm{Gd}(0001)$ thin film structure for Gd films grown on $\mathrm{Mo}(112)$ (Ref. 21) as well as the more unstrained $\mathrm{Gd}(0001)$ films grown on $\mathrm{W}(110){ }^{30}$

\section{B. Surface and bulk states of $\mathrm{Gd}(0001)$}

An important feature of the hcp Gd structure is the emergence of a relative gap at $E_{F}$ in the vicinity of the $\Gamma$ point and along the $\Gamma-A(\Delta)$ direction. This evidently leads to a rise in the gap in the projection of bulk bands onto the (0001) surface, which is essential for the appearance of true surface states near the center of the surface Brillouin zone. $^{21,29-31,53-56}$ Most of the calculated bulk bands are in good agreement with wave-vector (angle-resolved) photoemission and inverse photoemission experiments, as illustrated by the left panel (a) in Fig. 1, while the experimental bands near $E_{F}$, in the range from $\bar{\Gamma}$ to midway to the $\bar{M}$ point, occur within the gap and are acknowledged surface states. ${ }^{53-55}$

This is also true for the related bands observed with strained $\mathrm{Gd}(0001)$ grown on $\mathrm{Mo}(112)$ marked $C$ in the central panel (b) of Fig. 1. These bands ("C") are found to be sensitive to surface contamination and exhibit a conservation of the two dimensionality of the state (this is to say these bands do not show any noticeable dispersion with changing photon energy or $\mathbf{k}_{\perp}$ ), ${ }^{8,21}$ and therefore are attributed to surface-induced features. Hence, while the other bands $(A-G)$ may be related to specific projected bulk bands, bands $C$ appear within the gap in vicinity of the $\bar{\Gamma}$ point, which also indicates a surface origin of these states.

The band structure along $\bar{\Gamma}-\bar{M}(\bar{\Sigma}$ symmetry line in the surface Brillouin zone), calculated for a five-layer slab of $\mathrm{Gd}(0001)$ with a $4 \%$ expansive strained lattice, is presented in the right panel (c) of Fig. 1. Surface states and surface resonances for spin-majority and -minority electrons are marked by solid and dashed lines, respectively. True surface states, with more than $90 \%$ of the charge in the outmost surface layer, appear in the vicinity of $\bar{\Gamma}$ in the relative gap in the projected bulk band structure. This gap closes about midway to $\bar{M}$ along the high-symmetry line, and the surface states transform into surface resonances. Near $\bar{M}$, nevertheless, there appears to be another surface state located just under the bottom of the projected bulk bands.

By comparing calculated and experimental band structures presented in Fig. 1, the origin of most of the spectral peaks can be assigned. Thus the $C$ bands of strained $\mathrm{Gd}(0001)$ are obviously due to surface states which diminish the surface weight along $\bar{\Gamma}-\bar{M}$. Band $B$ [in Fig. 1(b)] is predominantly a bulk band with contributions from the surface resonances, which increase the surface weight near $\bar{M}$. Band $A$, that appears only for strained Gd films, ${ }^{8,21}$ unfortunately, cannot be directly explained in terms of initial states. This band may arise in angle-resolved photoemission because of possible influence of final states involving screening effects, in particular final-state $d-f$ mixing, ${ }^{59}$ and deserves further investigation, separate from the discussion herein.

The bands above $E_{F}, D, E$, and $G$ [in Fig. 1(b)] contain both surface and bulk contributions. The spin-majority bands $D$, above $E_{F}$, also appear to fall into the gap of the two projected bulk band structure near $\bar{\Gamma}$ and thus also may be attributed to a surface state near the Brillouin zone center.

\section{Density of states}

Shown in Fig. 2 are densities of states (DOS) for bulk hep Gd [Fig. 2(a)], the $\operatorname{Gd}(0001)$ surface [Fig. 2(b)], and the $\operatorname{Gd}(0001)$ surface with $4 \%$ expansive strain [Fig. 2(d)] that has been found for Gd films adsorbed on $\mathrm{Mo}(112){ }^{21}$ The most pronounced distinction between the bulk and surface DOS's is the appearance of the spin pair (spin-up and spindown) of high narrow peaks below $E_{F}$. Obviously, these peaks originate from the flat surface bands $C$ in strained $\operatorname{Gd}(0001)$ and their equivalent counterparts just above and below $E_{F}$ in unstrained $\operatorname{Gd}(0001)$. The strain causes a noticeable redistribution of heights for spin-up and spin-down surface peaks $(C)$ as well as for peaks above $E_{F}$. It should be noted that, in spite of narrowing bands, the width of the valence band below $E_{F}$ remains unchanged with the strain. This feature follows from a "pinning" of the Fermi level to the surface states which strongly contribute to the spectra.

There is a qualitative agreement between density of states for the bulk hcp Gd [Fig. 2(a)] and typical spin-resolved photoemission and inverse photoemission spectra for Gd(0001) films on $\mathrm{W}(110)$ at the $\bar{\Gamma}$ point $^{8}$ [Fig. 2(c)] and similarly for strained $\operatorname{Gd}(0001)$ films on $\operatorname{Mo}(112)$ at the $\bar{\Gamma}$ point $^{29}$ [Fig. 2(e)]. However, the spin-minority SPES peak just below $E_{F}$ does not find corresponding peak in bulk DOS's. Such a peak emerges from surface bands, as evidently follows from surface DOS's shown in Figs. 2(b) and 2(d). Worth noting are the changes in height and position of the bulk peaks at $-2 \mathrm{eV}$ [Fig. 2(a)] which, in surface DOS's [Fig. 2(b)], increases in binding energy to $-2.8 \mathrm{eV}$. On expansive strain, this pair of peaks further increases in height while their position stays almost the same.

Obviously, the surface DOS resembles the band structure integrated over entire surface Brillouin zone (BZ) while normal-emission photoemission spectra correspond to the $\bar{\Gamma}$ 

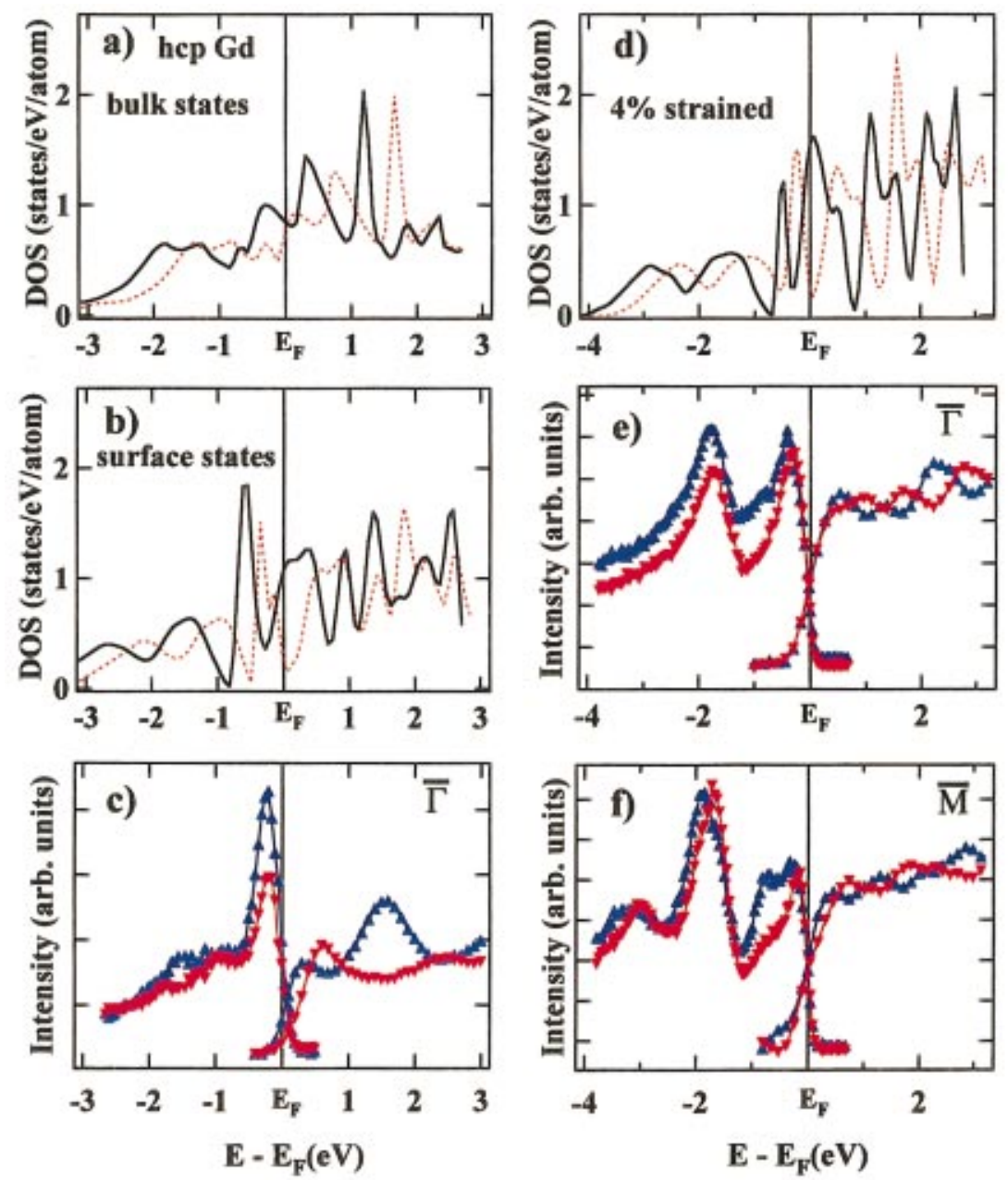

FIG. 2. (Color). The experimental results and theoretical calculations of density of states (DOS) for unstrained (on the left) and strained (on the right) $\mathrm{Gd}(0001)$ thin films. (a) and (b) are the calculated unstrained hcp $\operatorname{Gd}(0001)$ bulk and surface DOS's, respectively, with two spin majority (solid lines) and minority (dashed lines) indicated. (c) is the experimental results for spin polarized photoemission (Ref. 56) and inverse photoemission $^{31}$ of unstrained Gd(0001), grown on W(110), at the $\bar{\Gamma}$ point, with spin up (up ward triangle) and spin down (downward triangle) indicated (Ref. 8). The calculated surface DOS for $4 \%$ expanded strain $\mathrm{Gd}(0001)$ is shown in (d), with the theoretical DOS indicated according to the spin majority (solid lines) and spin minority (dashed lines). (e) and (f) are the experimental results of strained Gd, grown on a Mo(112) surface, with spin up (upward triangle) and spin down (downward triangle) at different symmetry points, (e) for the $\bar{\Gamma}$ point (f) for the $\bar{M}$ point of the surface Brillouin zone (Ref. 29). point. This can be the reason why the normal-emission $(\bar{\Gamma})$ photoemission peaks at $-2 \mathrm{eV}$ do not agree in binding energy with the surface DOS. It is well known that the surface sensitivity of the photoemission increases with increasing collection angle for photoelectrons, and these bands are well established to be very "bulklike" in character at normal emission in the unstrained $\operatorname{Gd}(0001)$ thin films. ${ }^{30}$ The peak in the surface DOS with energy $\left(E-E_{F}\right)$ below $-2 \mathrm{eV}$ originates from flat fragments of bands in vicinity of $\bar{M}$ (cf. Fig. 1 ), and therefore it is not surprising that angle-resolved photoemission (ARUPS) spectra, for strained $\mathrm{Gd}(0001)$ films, obtained for electron collection angle of $16^{\circ}$, which corresponds to the $\bar{M}$ point in the surface BZ [Fig. 2(f)], agree with the DOS calculated for strained Gd(0001) surface [Fig. 2(d)].

Not only do the bulk bands on the occupied side, well below $E_{F}(\sim-2 \mathrm{eV})$, change the spectral weight at $\bar{\Gamma}$ with increasing strain [Fig. 2(c) and 2(e)], but, as noted elsewhere, they also change symmetry. These bulk bands are of $\Delta_{1}$ (or $\left.a_{1}\right)$ symmetry $\left(d_{3 z^{2}-r^{2}}\right)$ in the unstrained $\operatorname{Gd}(0001)$, but of $\Delta_{5}, \Delta_{6}$ (or $\left.e_{1,2}\right)$ symmetry $\left(d_{x z}, d_{y z}\right)$ in strained $\operatorname{Gd}(0001) .{ }^{21}$ The possibility exists that the rare-earth band structure is perturbed by $f$ - $d$ and $f$-s hybridization (as previously suggested elsewhere ${ }^{60}$ ). This could be more pronounced with strain, due to the increasing overlap of $d$ and $f$ states with increasing localization. This would not be well modeled when the $4 f$ states are treated as core states. Such models exist, ${ }^{61,62}$ but are beyond the scope of this paper.

\section{Symmetry}

For $E$ and $G$ bands, separation of bulk and surface yields is somewhat involved because of close proximity of the bulk $d$ bands in this energy range, which makes the surface resonances rather weak. Nevertheless, the enhanced surface sensitivity of the inverse photoemission in combination with the agreement with theory permit some determination of symmetry and surface weight. Above the Fermi level, the DOS is strongly peaked while inverse photoemission spectroscopy (IPES) spectra, because of limited experimental resolution, are rather smooth, which complicates their analysis. To facilitate a comparison with experiment, the DOS's above $E_{F}$ presented in Fig. 3 are smoothed and arbitrarily scaled, while the spin-majority spectrum is decomposed into Lorentzian peaks, which are shown at the bottom of Fig. 3 by dotted lines.

In the vicinity of $\bar{\Gamma}$, the point group symmetries are the most restrictive and will have the greatest influence on the bonding hybridization possibilities. At the $\bar{\Gamma}$ point, the $C_{3 v}$ group symmetry (reduced from $C_{6 v}$ because of the hcp lattice packing) selects the states that can contribute to the IPES signal. Transitions from the free-electron state to the unoc- 


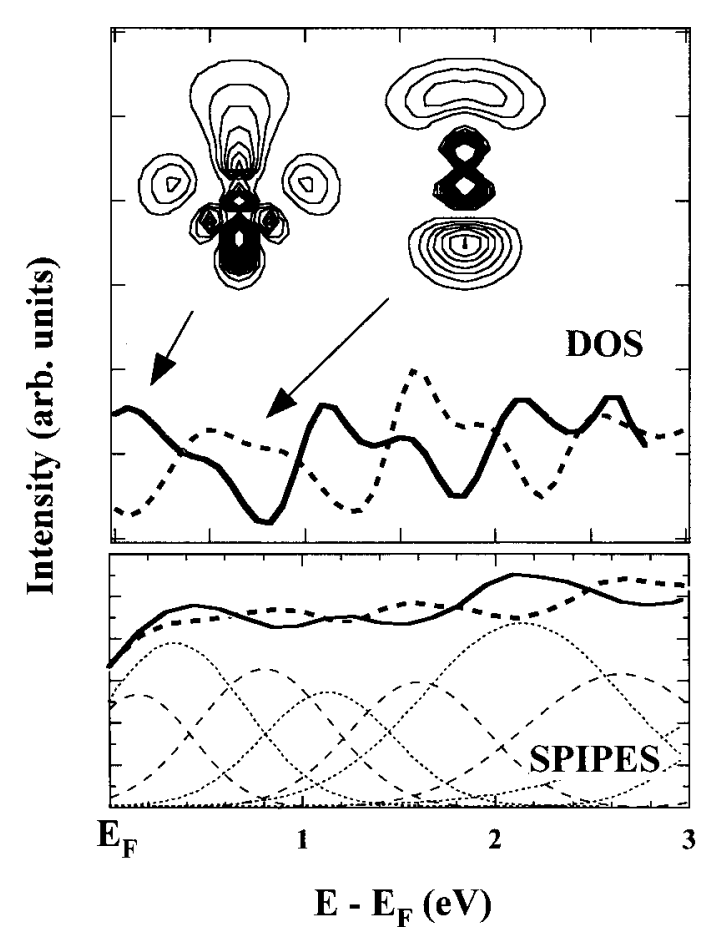

FIG. 3. The theoretical density of states above $E_{F}$ are compared with experiment. The theoretical DOS has been smoothed and arbitrary scaled to facilitate comparison with the SPIPES data. The spin-majority spectrum is decomposed into Lorentzian peaks which are shown by dotted lines. To illustrate symmetry of the surface states/resonances, the charge distributions (in the $X Z$ plane which is normal to the mirror plane) at $\bar{\Gamma}$ for two such states, located at the outmost surface atoms, are shown in the inset. The calculated spinminority surface state, at $+1.0 \mathrm{eV}$ at the $\bar{\Gamma}$ point, yields the peak $+0.7 \mathrm{eV}$ in the density of states which is of $d_{3 z^{2}-r^{2}}$ symmetry that is favorable for enhanced inverse photoemission. In contrast, the spin-majority surface resonance at $+0.7 \mathrm{eV}$ at the $\bar{\Gamma}$ point, which gives rise to the peak at $+0.3 \mathrm{eV}$, may be attributed to states with odd symmetry, so that the relative IPES peak is noticeably diminished.

cupied bound states of $d_{x y}$ states is forbidden by symmetry selection at $\bar{\Gamma}$, while transitions to the $d_{x^{2}-y^{2}}$ state are suppressed by the conservation of the $m_{j}$ rule. Thus transitions to the $p_{z}, p_{x}, p_{y}, d_{x z}, d_{y z}$, and $d_{3 z^{2}-r^{2}}$ symmetries are the dominant and almost exclusive contributions to the spectra in inverse photoemission.

To illustrate symmetry of the surface states/resonances, the charge distributions (in the $X Z$ plane which is normal to the mirror plane) at $\bar{\Gamma}$ for two such states, located at the outmost surface atoms, are shown in the inset in Fig. 3. Thus the spin-minority surface state at $+1 \mathrm{eV}$ at $\bar{\Gamma}$ is found to be predominantly of $d_{3 z^{2}-r^{2}}$ symmetry, so that it is favorable for enhanced inverse photoemission. In contrast, the spinmajority surface resonance at $+0.7 \mathrm{eV}$ at the $\bar{\Gamma}$ point, which gives rise to the peak in the total density of states at $+0.3 \mathrm{eV}$, may be attributed to the superposition of $p_{z}+d_{x z}+d_{x^{2}-y^{2}}$ and $d_{x y}$ states with odd symmetry, so that relative IPES peak is noticeably diminished, as can be seen in Fig. 3. This level ordering in the theoretical density of states for strained $\operatorname{Gd}(0001)$ is consistent with the level ordering, symmetry assignments, and roughly similar band separations for the unoccupied levels near $E_{F}$ obtained in resonant photoemission for relatively extensively strained (ultrathin) $\mathrm{Gd}(0001)$ on $\mathrm{W}(110)$ and $\mathrm{Gd}(0001)$ on $\mathrm{Cu}(100) .{ }^{63}$ The agreement between resonant photoemission and the total density of states is possible because resonant photoemission involving the unoccupied states is dominated by the integrated unoccupied density of states in the intermediate excitation, rather than by the wave vector.

Most of the bands found from ultraviolet photoemission spectroscopy and IPES studies for strained Gd(0001) films can be convincingly explained with respect to the bulk/ surface contribution which changes along $\overline{\Gamma M}$. The surface bands for strained $\mathrm{Gd}$ are found to be much similar to those for unstrained $\mathrm{Gd}(0001)$. This is not surprising because in experiment the main difference has been found for features that can be attributed to bulk.

\section{SUMMARY}

Most of the bands found from experimental wave-vectordependent spin-polarized band mapping studies for strained $\operatorname{Gd}(0001)$ films qualitatively resemble the theoretical band structure. The relative bulk/surface contributions along $\bar{\Gamma}-\bar{M}$ can be convincingly assigned on this basis. The surface bands for strained $\mathrm{Gd}$ are found to be very similar to those found for unstrained $\mathrm{Gd}(0001)$. Expansive strain does result in an increase in the exchange splitting and increased localization both in experiment and theory, particularly at the surface.

The results of this comparison do suggest a possible $d-f$ hybridization in either the photoemission initial state or final state, affecting the bottom of the valence band to an appreciable extent. The unoccupied bands for strained $\operatorname{Gd}(0001)$ show the characteristic dispersion predicted by theory and a number of unoccupied states have been identified, which was not previously possible.

Not only is this work consistent with the influence of strain in rare-earth alloys, ${ }^{19,20}$ but similar strain effects are suggested by the work in rare-earth compounds. The extent of in-plane strain is clearly seen to affect magnetic properties of the manganese perovskites $\mathrm{La}_{1-x} A_{x} \mathrm{MnO}_{3} .{ }^{9-17}$ These are also local moment ferromagnetic systems. Unfortunately, the band structure must take into account a realistic model of the surface band structure and surface composition in such systems, which can be quite complex in alloys and perovskites, ${ }^{64-68}$ so that a comparison, similar to the one presented here, between the experimental band structures in the perovskite systems with theory presents a very significant challenge.

\section{ACKNOWLEDGMENTS}

This work was supported by the NSF through Grant Nos. DMR-94-07933 and DMR-98-02126, the Center for Materials Research and Analysis (CMRA), and the Nebraska Research Initiative at the University of Nebraska, and the North 
Atlantic Treaty Organization (NATO) under Grant No. PST.CLG.976845. The spin-polarized photoemission experiments were carried out at the National Synchrotron Light Source which is funded by the U.S. DOE. The authors would like to acknowledge the assistance of E. Vescovo, C. Waldfried, T. Rybnicek, and D. McIlroy for their assistance with parts of the measurements reported herein, and acknowledge some helpful discussions with Bruce Harmon.
${ }^{1}$ V. L. Moruzzi, P. M. Marcus, K. Schwarz, and P. Mohn, Phys. Rev. B 34, 1784 (1986).

${ }^{2}$ S. R. Shinde, R. Ramesh, S. E. Lofland, S. M. Bhagat, S. B. Ogale, R. P. Sharma, and T. Venkatesan, Appl. Phys. Lett. 72, 3443 (1998).

${ }^{3}$ H. Bartholin, J. Beille, D. Bloch, P. Boutron, and J. L. Feron, J. Appl. Phys. 42, 1679 (1971).

${ }^{4}$ U. Enz, Physica (Amsterdam) 26, 698 (1960).

${ }^{5}$ B. R. Cooper, Solid State Phys. 21, 393 (1968).

${ }^{6}$ R. G. Chapman and N. H. March, J. Magn. Magn. Mater. 61, 81 (1986).

${ }^{7}$ C. Waldfried, T. McAvoy, D. Welipitiya, P. A. Dowben, and E. Vescovo, Europhys. Lett. 42, 685 (1998).

${ }^{8}$ C. Waldfried, T. McAvoy, D. Welipitiya, T. Komesu, P. A. Dowben, and E. Vescovo, Phys. Rev. B 58, 7434 (1999).

${ }^{9}$ Y. Moritomo, A. Asamitsu, and Y. Tokura, Phys. Rev. B 51, 16491 (1995).

${ }^{10}$ Y. Suzuki, H. Y. Hwang, S.-W. Cheong, and R. B. van Dover, Appl. Phys. Lett. 71, 140 (1997).

${ }^{11}$ C. Kwon, M. C. Robson, K.-C. Kim, J. Y. Gu, S. E. Lofland, S. M. Bhagat, Z. Trajanovic, M. Rajeswari, T. Venkatesan, A. R. Krafz, R. D. Gomez, and R. Ramesh, J. Magn. Magn. Mater. 172, 229 (1997); S. E. Lofland, S. M. Bhagat, H. L. Ju, G. C. Xiong, and T. Venkatesan, J. Appl. Phys. 79, 5166 (1996).

${ }^{12}$ M. Izumi, Y. Konishi, T. Nishihara, S. Hayashi, M. Shinohara, M. Kawasaki, and Y. Tokura, Appl. Phys. Lett. 73, 2497 (1998); Y. Konishi, Z. Fang, M. Izumi, T. Manako, M. Kasai, H. Kuwahara, M. Kawasaki, K. Terakura, and Y. Tokura, J. Phys. Soc. Jpn. 68, 3790 (1999); Y. Ogimoto, M. Izumi, T. Manako, T. Kimura, Y. Tomioka, M. Kawasaki, and Y. Tokura, Appl. Phys. Lett. 78, 3505 (2001).

${ }^{13}$ J. O’Donnell, M. S. Rzchowski, J. N. Eckstein, and I. Bozovic, Appl. Phys. Lett. 72, 1775 (1998); X. W. Wu, M. S. Rzchowski, H. S. Wang, and Qi. Li, Phys. Rev. B 61, 501 (2000).

${ }^{14}$ H. S. Wang, Qi. Li, Kai Liu, and C. L. Chien, Appl. Phys. Lett. 74, 2212 (1999).

${ }^{15}$ W. Prellier, A. M. Haghiri-Gosnet, B. Mercey, P. Lecoeur, M. Hervieu, C. Simon, and B. Raveau, Appl. Phys. Lett. 77, 1023 (2000).

${ }^{16}$ F. Tsui, M. C. Smoak, T. K. Nath, and C. B. Eom, Appl. Phys. Lett. 76, 2421 (2000); R. A. Rao, D. Lavric, T. K. Nath, C. B. Eom, L. Wu, and F. Tsui, J. Appl. Phys. 85, 4794 (1999); T. K. Nath, R. A. Rao, D. Lavric, C. B. Eom, L. Wu, and F. Tsui, Appl. Phys. Lett. 74, 1615 (1999).

${ }^{17}$ J. Zhang, H. Tanaksa, T. Kanki, J. H. Choi, and T. Kawai, Phys. Rev. B 64, 184404 (2001); J. Zhang, H. Tanaka, and T. Kawai, J. Appl. Phys. 90, 6275 (2001); T. Kanki, H. Tanaka, and T. Kawai, Phys. Rev. B 64, 224418 (2001).

${ }^{18}$ H. M. Fretwell et al., Phys. Rev. Lett. 82, 3867 (1999).

${ }^{19}$ A. Andrianov, Pis'ma Zh. Eksp. Teor. Fiz. 55, 639 (1992) [JETP Lett. 55, 666 (1992)].
${ }^{20}$ A. Andrianov, J. Magn. Magn. Mater. 140-144, 749 (1995).

${ }^{21}$ C. Waldfried, D. N. McIlroy, and P. A. Dowben, J. Phys.: Condens. Matter 9, 10615 (1997).

${ }^{22}$ P. A. Dowben, S. Varma, Y. J. Kime, D. R. Mueller, and M. Onellion, Z. Phys. B: Condens. Matter 73, 247 (1988).

${ }^{23}$ N. K. Singh, P. Dale, D. Bullett, and R. G. Jones, Surf. Sci. 294, 333 (1994).

${ }^{24}$ I. N. Yakovkin, Surf. Sci. 406, 57 (1998).

${ }^{25}$ H. J. F. Jansen, A. J. Freeman, M. Weinert, and E. Wimmer, Phys. Rev. B 28, 593 (1983).

${ }^{26}$ I. N. Yakovkin, Surf. Sci. 488, 7 (2001).

${ }^{27}$ D. Weller and S. F. Alvarado, J. Appl. Phys. 59, 2908 (1986).

${ }^{28}$ Takashi Komesu, C. Waldfried, and P. A. Dowben, IEEE Trans. Magn. 36, 2915 (2000).

${ }^{29}$ Takashi Komesu, C. Waldfried, and P. A. Dowben, Phys. Lett. A 256, 81 (1999).

${ }^{30}$ P. A. Dowben, D. N. McIlroy, and Dongqi Li, in Handbook on the Physics and Chemistry of Rare Earths, edited by K. A. Gschneidner and LeRoy Eyring (North Holland, Amsterdam, 1997) Vol. 24, Chap. 159 (1997) pp. 1-46; Magnetism and Electronic Correlations in Local Moment Systems, edited by M. Donath, P. A. Dowben and W. Nolting (World Scientific, Singapore, 1998), ISBN 981-02-3538-0.

${ }^{31}$ M. Donath, B. Gubanka, and F. Passek, Phys. Rev. Lett. 77, 5138 (1996).

${ }^{32}$ M. Donath, J. Phys.: Condens. Matter 11, 9421 (1999).

${ }^{33}$ V. I. Anisimov, J. Zaanen, and O. K. Andersen, Phys. Rev. B 44, 943 (1991).

${ }^{34}$ D. M. Bylander and L. Kleinman, Phys. Rev. B 50, 1363 (1994).

${ }^{35}$ D. M. Bylander and L. Kleinman, Phys. Rev. B 49, 1608 (1994); J. Morrison, D. M. Bylander, and L. Kleinman, Phys. Rev. Lett. 71, 1083 (1993).

${ }^{36}$ O. Eriksson, R. Ahuja, A. Ormeci, J. Tregg, O. Hjorstam, P. Soderlind, B. Johansson, and J. M. Willis, Phys. Rev. B 52, 4420 (1995).

${ }^{37}$ B. N. Harmon and A. J. Freeman, Phys. Rev. B 10, 1979 (1974).

${ }^{38}$ B. N. Harmon, V. P. Antropov, A. I. Liechtenstein, I. V. Solovyev, and V. I. Anisimov, J. Phys. Chem. Solids 56, 1521 (1995).

${ }^{39}$ M. Heinemann and W. M. Temmerman, Phys. Rev. B 49, 4348 (1994).

${ }^{40}$ J. F. Herbst, D. N. Lowy, and R. E. Watson, Phys. Rev. B 6, 1913 (1972).

${ }^{41}$ R. F. Sabirianov and S. S. Jaswal, Phys. Rev. B 55, 4117 (1997).

${ }^{42}$ A. B. Shick, A. I. Liechtenstein, and W. E. Pickett, Phys. Rev. B 60, 10763 (1999).

${ }^{43}$ A. B. Shick, W. E. Pickett, and C. S. Fadley, Phys. Rev. B 61, R9213 (2000).

${ }^{44}$ D. J. Singh, Phys. Rev. B 44, 7451 (1991).

${ }^{45}$ R. Wu, C. Li, A. J. Freeman, and C. L. Fu, Phys. Rev. B 44, 9400 (1991). 
${ }^{46}$ H. Krakauer, M. Posternak, and A.-J. Freeman, Phys. Rev. B 19, 1706 (1979).

${ }^{47}$ M. Posternak, H. Krakauer, A. J. Freeman, and D. D. Koelling, Phys. Rev. B 21, 5601 (1980).

${ }^{48}$ H. J. F. Jansen, A. J. Freeman, M. Weinert, and E. Wimmer, Phys. Rev. B 28, 593 (1983).

${ }^{49}$ O. Jepsen, J. Madsen, and O. K. Andersen, Phys. Rev. B 18, 605 (1978).

${ }^{50}$ F. Ciccacci, H.-J. Drouhin, C. Hermann, R. Houdre, and G. Lampel, Appl. Phys. Lett. 54, 632 (1989).

${ }^{51}$ P. D. Johnson et al., Rev. Sci. Instrum. 63, 1902 (1992).

${ }^{52}$ Dongqi Li, P. A. Dowben, J. E. Ortega, and F. J. Himpsel, Phys. Rev. B 49, 7734 (1994).

${ }^{53}$ Dongqi Li, C. W. Hutchings, P. A. Dowben, C. Hwang, RongTzong Wu, M. Onellion, A. B. Andrews, and J. L. Erskine, J. Magn. Magn. Mater. 99, 85 (1991).

${ }^{54}$ R. q. Wu and A. J. Freeman, J. Magn. Magn. Mater. 99, 81 (1991).

${ }^{55}$ D. Li, J. Zhang, P. A. Dowben, and M. Onellion, Phys. Rev. B 45, 7272 (1992).

${ }^{56}$ Dongqi Li, J. Pearson, S. D. Bader, D. N. McIlroy, C. Waldfried, and P. A. Dowben, Phys. Rev. B 51, 13895 (1995).
${ }^{57}$ R. Hott, Phys. Rev. B 44, 1057 (1991).

${ }^{58}$ J. S.-Y. Wang and M. Rasolt, Phys. Rev. B 13, 5330 (1976).

${ }^{59}$ J. E. Ortega, F. J. Himpsel, Dongqi Li, and P. A. Dowben, Solid State Commun. 91, 807 (1994).

${ }^{60}$ P. Strange, A. Svane, W. M. Temmerman, Z. Szotek, and H. Winter, Nature (London) 399, 756 (1999).

${ }^{61}$ S. M. Jaya and W. Nolting, Physica B 292, 359 (2000).

${ }^{62}$ G. G. Reddy, D. Meyer, S. Schwieger, A. Ramakanth, and W. Nolting, J. Phys.: Condens. Matter 12, 7463 (2000).

${ }^{63}$ P. A. Dowben, Dongqi Li, Jiandi Zhang, and M. Onellion, J. Vac. Sci. Technol. A 13, 1549 (1995).

${ }^{64}$ Jaewu Choi, C. Waldfried, S.-H. Liou, and P. A. Dowben, J. Vac. Sci. Technol. A 16, 2950 (1998).

${ }^{65}$ Jaewu Choi, Jiandi Zhang, S.-H. Liou, P. A. Dowben, and E. W. Plummer, Phys. Rev. B 59, 13453 (1999).

${ }^{66}$ Hani Dulli, E. W. Plummer, P. A. Dowben, Jaewu Choi, and S.-H. Liou, Appl. Phys. Lett. 77, 570 (2000).

${ }^{67}$ Hani Dulli, P. A. Dowben, S.-H. Liou, and E. W. Plummer, Phys. Rev. B 62, R14 629 (2000).

${ }^{68}$ C. N. Borca, Bo Xu, Takashi Komesu, Hae-Kyung Jeong, M. T. Liu, S.-H. Liou, and P. A. Dowben, Surf. Sci. Lett. 512, L346 (2002). 